\title{
The Psychosocial Approach to Rehabilitation of the Spinal Cord Injured Patient
}

\author{
F. K. Judd, MD, DPM, FRANZCP, ${ }^{1}$ D. J. Brown, MBBS, FRACP ${ }^{2}$ \\ ${ }^{1}$ Liaison Psychiatrist, ${ }^{2}$ Medical Director, Spinal Injuries Unit, Austin Hospital, \\ Heidelberg, Vic. 3084, Australia.
}

\begin{abstract}
Summary
The development of a liaison psychiatry service to the Austin Hospital Spinal Injuries Unit highlighted the need for a psychosocial approach to patients rehabilitation. Increasingly we recognised that psychological factors influenced the course of the patient's acute illness and rehabilitation; this led to the formation of a psychosocial group under the leadership of the liaison psychiatrist. Membership of this group includes the liaison psychiatrist, psychiatry registrar, clinical psychologist, social workers, visiting nurse, chaplains and the rehabilitation registrar.

The psychosocial approach advocates the consideration of psychosocial management in all aspects of patient care. In this paper we describe the structure and function of the psychosocial team in the management of patients in the Spinal Injuries Unit at the Austin Hospital.
\end{abstract}

Key words: Spinal injuries; Psychosocial management; Rehabilitation.

Rehabilitation of spinal cord injured people has traditionally emphasised physical rehabilitation and relied upon support and empathy from staff members to assist patients with their psychological and social difficulties. Of recent times social workers have provided more specialised counselling, but generally their number has been limited so that the service provided has been thinly spread. Such was the situation in the early years of our Spinal Unit after its establishment in 1957. More specialised help, on a consultation basis, was sought from psychiatrists only for the treatment of floridly psychotic patients. Recently the needs for better assessment of patients and for training of and support of staff led to the appointment of a liaison psychiatrist whose responsibilities include the Spinal Injuries Unit and of a senior psychiatry registrar working full time in the Unit. It has thus proved possible to foster the psychosocial rehabilitation of patients in a more expert way, utilising the additional skills of highly trained personnel and enabling the training of staff to greater awareness and competence in this area of rehabilitation. The appointment of a part-time clinical psychologist has extended the range of treatment options available to patients. 
While it was clear that the psychiatrist was the most appropriate person for psychotherapeutic management of patients, and to treat overtly psychotic patients, it was less clear which staff member could best handle other situations. Overlap in skills between chaplain, social workers, clinical psychologist and psychiatrist had the potential to lead to poor management of patients who might be seen for the same problem by people from different disciplines. Also some patients might receive much attention, whilst others remain neglected. In order to co-ordinate the counselling services available to patients so that each patient received help most appropriate to his/her particular difficulties and problems of adjustment, we needed to develop a system whereby patients could be assessed and then followed up by the person with the most appropriate skills. It was also necessary to develop inservice training for staff members in nursing and allied health groups who are often the major effectors of treatment. Such staff members required supervision by someone with specific training in counselling. The psychosocial group was therefore formally organised for the express purpose of assessing the need for and determining the most appropriate treatment, deciding which member of the group was most appropriate to treat, either directly or indirectly a patient, and also to provide skilled supervision of those involved in counselling. We have previously reviewed the role of psychiatry in the Spinal Injuries Unit (Judd and Burrows, 1986; Judd and Brown, 1987). We now describe the structure and function of the psychosocial group. To our knowledge this has not been previously described in the rehabilitation of spinal cord injured patients.

\section{The psychosocial group}

The liaison psychiatrist, skilled in medicine, neurochemical and neuropharmacological concepts and with a knowledge of psychosocial issues leads the group. All new admissions to the SIU are assessed at the earliest opportunity by the liaison psychiatrist. This early assessment focuses on determining the individual's personality structure and vulnerabilities, habitual means of coping with stress and capacity to deal successfully with his/her disability. Individual liability to discrete psychiatric disorder is also evaluated. This assessment facilitates the planning of services to assist patients 'at risk' for poor outcome, and of measures to assist staff to deal with those patients likely to be non compliant and non co-operative with treatment. In addition it provides a basis for optimal rehabilitation planning, information to assist other members to understand the patient and his/her reactions and for subsequent assessment and treatment should discrete psychological or behavioural problems develop. The psychiatrist is usually the most appropriate member of the group to assess patients where specific psychological disorders are identified. As with any medically ill patient, we must ensure that organically based psychopathology is identified and the causative factors appropriately treated. Where organic factors are excluded, various forms of psychotherapy or pharmacotherapy may be indicated. Various members of the group may take part in the psychotherapeutic management of patients. Therapeutic skills provided by the psychiatrist include various forms of individual and group psychotherapy, family therapy and pharmacotherapy.

The work of the clinical psychologist complements that of the psychiatrist. 
Specific assessment skills may include psychological and neuropsychological testing. Treatment strategies such as cognitive therapy, various forms of behavioural therapy, social skills and assertiveness training, relaxation therapy and hypnosis may be utilised.

The social worker assists patients and their families with normal emotional/psychological reactions to their injury, to adapt to disability and to reestablish competencies. Specific areas of involvement include problems with formal organisations, e.g. accommodation, legal problems, resolution of interpersonal conflict and problems with social relationships and role performance and adaptation to loss of function, income, accommodation and resources.

The home visiting nurse and rehabilitation registrar attend the meeting to discuss patients with whom they encounter particular problems and to provide information and feedback to other members of the group. Participation in the group provides the opportunity for education and supervision of psychosocial treatment strategies.

The chaplains provide pastoral care allowing expression of faith, giving support and acting as the religious conscience of the institution. Although they do not provide formal psychological therapy they attend the psychosocial group. The chaplains gain knowledge and understanding of various psychological and behavioural problems by their participation as well as contributing valuable information about various patients.

Weekly group meetings are held which have several functions. Requests from other team members for psychological assessment and treatment are evaluated at the meeting and a member of the team designated to see each patient. All such patient assessments are reviewed at the next meeting. In addition patients and families who are seen by various members of the group are discussed each week. At these meetings various clinical problems and treatment strategies are discussed. This meeting provides an opportunity for the complementary use of various forms of expertise and clinical supervision under the leadership of the liaison psychiatrist. The integration of physical and psychosocial therapies is essential to successful implementation of the biopsychosocial approach. In addition to the weekly meetings, members of the psychosocial group attend ward rounds, team meetings, rehabilitation and family meetings along with other members of the treatment team. Regular liaison between the medical director of the unit and the leader of the psychosocial group has facilitated this integration.

\section{The psychosocial management}

\section{The patient and his family}

The biopsychosocial approach advocates the consideration of psychosocial management in all aspects of patient care (Lipowski, 1974). The general aims of contact with spinal cord injured patients include the promotion of normal adaptation to loss, facilitation of the family's adaptation to their changed circumstances, maximising remaining abilities and the development of new abilities.

Individual psychotherapy for patients may be provided by a member of the psychosocial group or by any other team member with supervision from a member of the group. At times, the nature of individual patient problems is 
such that all of the patient's rehabilitation team become involved in his/her psychological management.

Therapy is available to all patients to assist in coping with the multiple losses experienced to facilitate the normal adaptation process. Therapy utilises the principles of grief work and supportive psychotherapy and aims to promote the patient's best psychological and social functioning by restoring and reinforcing his/her abilities to manage life. Therapy may include reassurance, particularly the removal of doubts and misconceptions and emphasis of the individual's assets, explanation, focused on day to day practical questions, making clearer the nature of the problems which the patient faces, direct advice; suggestion, explicit or implicit, encouragement and bolstering of self-esteem, effecting changes in the patients environment and encouraging catharsis. The individual may need support in the expression of affects aroused by the injury, such as anger, sadness, and guilt with additional support during recapitulation of memories and feelings associated with a lifestyle which must now change.

At the first evaluation, particular care must be taken to identify those patients 'at risk' for the development of a depressive illness or other manifestations of failure of adaptation to their injury. Such patients need regular review to allow early detection and treatment of these problems. A patient's past or family history of depression, previous personal difficulty coping with loss, difficulty with interpersonal relationships, lack of support and the pressure of concurrent crisis may all increase the risk of depression. Where the injury interferes with the patient's coping strategies or ability to maintain self-esteem, particular difficulty in adapting may be experienced (Judd et al., 1986).

Patients with a past history of psychotic illness and those known to abuse alcohol or drugs need regular attention by the psychiatrist or clinical psychologist for management of these problems, all of which may be exacerbated by the stress of the injury and hospitalisation.

The patient's family should also be evaluated at the time of initial assessment, again intervention aims to promote adaptation to the injured member's disability. Both short term adjustment and long term adaptation to the injury are considered. Particular care is paid to the needs of all family members though priority is given to the injured member. Family or marital therapy may prove valuable where pathological family interactional patterns are identified and in the presence of relationship difficulties. When the children of spinal injured parents show difficulties in coping, individual or family therapy is utilised.

The many facets of sexual counselling are shared between members of the psychosocial team. All patients are seen by the visiting nurse before discharge and the practical aspects of sexual activity discussed. This aspect of sexual counselling is then readily reviewed after discharge. Relationship issues are generally discussed with the patient's primary therapist.

Problems which may lead to specific review and re-evaluation during rehabilitation include somatic complaints for which no organic cause is found, change in habitual behaviour, the development of identifiable psychiatric disorder and various forms of deviant illness behaviour, e.g. non compliance, gross denial of illness, self-destructive behaviour including suicide attempts and excess dependency. When review is requested for such problems, the patient is discussed at the psychosocial team meeting and a member of the team de- 
signated to assess the patient. Where the cause of the problem is unknown, when an organic problem is suspected or where the request is in response to the onset of a discrete psychiatric illness, the psychiatrist undertakes the diagnostic evaluation. Subsequently, with the clarification of the origin and nature of the problem we may involve other members of the psychosocial team in the treatment or the supervision of others in the management of the patient and perhaps his family and the wider social milieu.

\section{The staff}

When the psychosocial group was first formed we focused our activities on developing our own knowledge and skills and forming a cohesive team whose members' expertise complimented each other. Having achieved this task we began working towards our second goal: that of developing a biopsychosocial orientation among the wider treating team. This has involved two main activities: weekly ward meetings with nursing staff, and where possible with other paramedical staff. These meetings ususally focus on 'the problem patient', with discussion about understanding the causes of the patient's behaviour, symptoms and emotional responses and strategies for dealing with those patients who distress staff or other patients. They also allow staff to ventilate their own feelings of anger, sadness, guilt and frustration and to find reassurance that these feelings are natural and acceptable. Guidelines for personal interaction with patients including limit setting may be discussed in this forum.

Secondly, members of the psychosocial team participate in the inservice training programme along with other members of the unit. At this time, formal education may be provided on many topics including the major psychiatric disorders, the psychosocial consequences of physical illness, family functioning and pathology, and stress management. Of course, this programme also enables the psychosocial team to improve their own knowledge of the medical and paramedical physical care of the spinal cord injured. This integration of knowledge of the physical and psychosocial aspects of patient care is essential to the biopsychosocial approach.

\section{After discharge}

Psychosocial care of the patient and his family af ter discharge is maintained in three major ways. Firstly, when attending routine outpatient follow-up after initial discharge, patients are seen by the psychiatrist as well as the medical and paramedical staff. Secondly, in addition to attending the weekly psychosocial meeting, the visiting nurse meets the psychiatrist regularly for supervision and this enables the psychiatrist to provide guidance and support to the visiting nurse who may then act as a primary therapist for either the patient and/or his family. When particular problems are identified, the psychiatrist may review the patient and organise ongoing treatment as appropriate. Thirdly the psychiatrist provides follow-up via secondary consultation to a number of agencies, e.g. the patient's local doctor and nursing home staff. Of course, patients returning to the hospital for any number of identified problems may be seen for psychiatric reassessment as requested. Those patients previously identified as being 
'at risk' and those who have required specific psychosocial treatments during rehabilitation are seen regularly following discharge. Where practicable such follow-up is provided by the therapist who has seen the patient during their inpatient stay. Where, for example, geography makes this impractical, local follow-up is arranged prior to discharge.

\section{Conclusion}

The development of a psychosocial group in the rehabilitation of spinal cord injuries must be seen in the same light as the physiotherapist and occupational therapist group and the medical/nursing group, which form part of the overall management team working together towards the rehabilitation of the patient with spinal cord injury. The organisational functioning of the group as described above enables both patient and family to have the best access to appropriate counselling services, provides training and development for other members of the treating team, optimises the use of the available resources and serves to highlight the importance of this area of rehabilitation. It has been a useful way of stimulating research in order to prevent the development of more severe and catostrophic psychosocial problems such as severe depression.

\section{References}

JUdD FK, BRown DJ 1987 Psychiatry in the spinal injuries unit. Paraplegia 25:254-257.

JudD FK, BURrows GD 1986 Liaison psychiatry in a spinal injuries unit. Paraplegia 24:6-19.

JuDD FK, BurRows GD, BRown DJ 1986 Depression following acute spinal cord injury. Paraplegia 24:358-364.

LIPOWSKI ZJ 1974. Consultation-liaison psychiatry: an overview. American fournal of Psychiatry 131:623-630. 